\title{
Approximate Rotational Symmetries in Electron Nanodiffraction from Amorphous Materials
}

\author{
Shuoyuan Huang, Carter Frances, Paul M. Voyles
}

Department of Materials Science and Engineering, University of Wisconsin-Madison, 1509 University Ave, Madison WI 53705, USA

$$
\text { paul.voyles@wisc.edu }
$$

Although amorphous materials lack long-range translation order, they are strongly ordered at the length scale of single interatomic bonds and retain some order into more distant coordination shells. One form of order is local, approximate rotational symmetry. For example, local five-fold rotational symmetry has been shown in simulations to correlate to slower dynamics in metallic liquids and greater strength in metallic glasses [1]. Local structural symmetry gives rise to symmetry in electron nanodiffraction speckle patterns acquired with sub-nanometer probes but evaluating symmetries in patterns from amorphous materials is challenging. Only small clusters of atoms have symmetry, their symmetry is often distorted or imperfect, and the symmetric cluster is embedded in more atoms which are disordered. One method to assess symmetries in nanodiffraction is the angular power spectrum. We have used angular power spectrum data to demonstrate that $\mathrm{Zr}_{65} \mathrm{Cu}_{27.5} \mathrm{Al}_{7.5}$ glasses exhibit increasing 4-fold and 5-fold structural symmetry with increasing stability in the glassy state and increasing hardness [2].

However, angular symmetry is subject to three artifacts that give rise to power that does not correspond to symmetries in the structure. First, aliasing transfers power from lower $n$ to higher $n$. Second, electron nanodiffraction patterns without Friedel symmetry give rise to non-structural odd-order power. Third, the extra atoms surrounding the ordered cluster can create speckles which cause the power in rotational orders that are not present in the structure. We have proposed a different method to assess symmetries in amorphous nanodiffraction inspired by the Symmetry STEM method for crystals [3]. This method defines symmetry coefficients $S_{n}$ which sample only the discrete angles associated with $n$-fold symmetry [4]. The discrete sampling avoids the aliasing and Friedel breakdown artifacts entirely, and it reduces the incidence of the structure overlap artifact. Electron scattering simulations in Figure 1(a) show that $S_{n}$ is sensitive to nearest-neighbour icosahedral order in metallic glass atomic models. Experiments on $\mathrm{Pd}_{43} \mathrm{Ni}_{10} \mathrm{Cu}_{27} \mathrm{P}_{20}$ in $\mathrm{Figure}_{1}(\mathrm{~b})$ result in symmetries consistent with dodecahedral structure found in previous studies.

Figure 1(c) demonstrates the use of symmetry coefficients for spatial mapping of high-symmetry clusters. It is derived from a 4D STEM data set acquired with a high sensitivity, high-speed direct electron detection camera recently developed by the Wisconsin Materials Research Science and Engineering Center and Direct Electron, Inc. These data were acquired at 7,000 frames per second (fps). The current maximum speed of the camera is $24,000 \mathrm{fps}$, and the ultimate design speed is in excess of $100,000 \mathrm{fps}$. These extremely high speed will enable time-resolved, in situ experiments using symmetry coefficients to track the evolution of local symmetries in supercooled liquids as they cool through the glass transition.
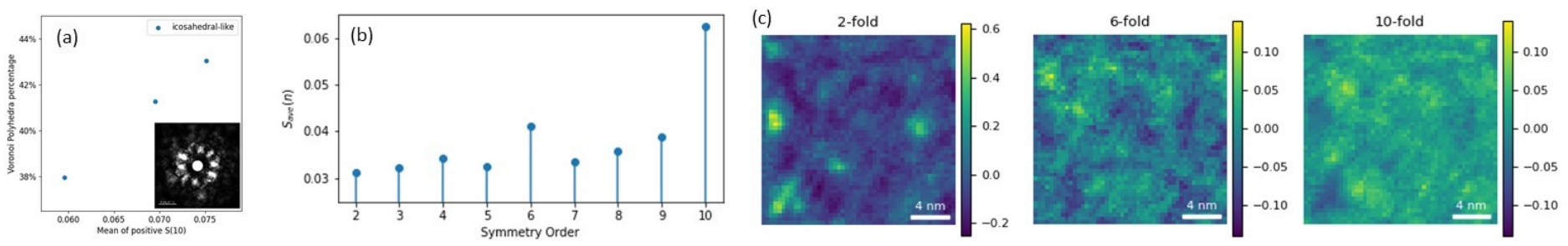

Figure 1. (a) $S_{10}$ as a function of a measure of the icosahedral order in atomic models of Al-Sm metallic glass. (b) Average $S_{n}$ for $\mathrm{Pd}_{43} \mathrm{Ni}_{10} \mathrm{Cu}_{27} \mathrm{P}_{20}$ metallic glass. (c) A map of 2-, 4-, 6-, and 10-fold symmetry from Pt metallic glass nanowires.

[1] Cheng, Y. Q. Q., Ma, E. (2011) Prog. Mater. Sci. 56, 379.

[2] Muley, S. V., Cao, C., Chatterjee, D., Francis, C., Lu, F. P., Ediger, M. D., Perepezko, J. H., Voyles, P. M. (2021). Phys. Rev. Mater. 5, 033602.

[3] Krajnak, M. \& Etheridge, J. A (2020) Proc. Natl. Acad. Sci. 117, 27805.

[4] Shuoyuan, H., Francis, C., Ketkaew J., Schroers J., Voyles P. M. (in preparation)

Keywords: electron nanodiffraction; rotational symmetry; amorphous materials; direct electron camera

This research was supported by the US National Science Foundation (DMR-1807241). Development of the ultrafast TEM camera was supported by the Wisconsin MRSEC (DMR-1720415).

Acta Cryst. (2021), A77, C372 